\title{
CEuvrer à la réconciliation par l'éducation : Juifs et Palestiniens en Israël
}

Working for reconciliation through education: Jews and Palestinians in Israel Obrar por la reconciliación mediante la educación: Judíos y Palestinos en Israel

\section{Zvi Bekerman}

Traducteur : Sylvaine Herold

\section{OpenEdition}

\section{Journals}

Édition électronique

URL : https://journals.openedition.org/ries/6498

DOI : $10.4000 /$ ries.6498

ISSN : 2261-4265

Éditeur

France Education international

Édition imprimée

Date de publication : 1 septembre 2018

Pagination : 67-78

ISBN : 978-2-85420-619-7

ISSN : $1254-4590$

\section{Référence électronique}

Zvi Bekerman, « EEuvrer à la réconciliation par l'éducation : Juifs et Palestiniens en Israël », Revue

internationale d'éducation de Sèvres [En ligne], 78 । septembre 2018, mis en ligne le 01 septembre 2020, consulté le 25 juin 2021. URL : http://journals.openedition.org/ries/6498 ; DOI : https://doi.org/

$10.4000 /$ ries. 6498 


\title{
Euvrer \\ à la réconciliation par l'éducation : Juifs et Palestiniens en Israël*
}

\author{
Zvi Bekerman \\ Université hébraïque de Jérusalem
}

Le manque de cohésion sociale est considéré de nos jours comme l'un des problèmes majeurs dont souffrent les sociétés occidentales. Il génère instabilité sociale et conflits, menaçant ainsi la stabilité nationale comme internationale. Le manque de cohésion sociale est surtout constaté parmi les groupes considérés comme appartenant à des cultures clairement différenciées. Leurs différences culturelles formeraient la base de tensions sociales et de conflits territoriaux. L'éducation figure, au premier rang des solutions envisagées par les gouvernements et les organisations internationales, comme l'un des principaux domaines où le manque de cohésion sociale devrait être traité. On attend de l'école qu'elle aide à dépasser les divisions sociales pour contribuer à la stabilité et au développement pacifique de la société. Les discours éducatifs insistent surtout sur les stratégies d'intégration, qui seraient les plus adaptées pour tenter de surmonter les tensions sociétales. Dans cet article, nous passerons brièvement en revue les politiques d'intégration mises en œuvre en Israël, qui consistent finalement, pour l'essentiel, en des tentatives plutôt mineures d'intégration réciproque entre les citoyens juifs et palestiniens d'Israël. Bien que la rhétorique de l'intégration englobe à la fois l'éducation ethnique/nationale et l'éducation spéciale, ces deux voies sont en grande partie prises en compte séparément dans le discours éducatif israélien.

\section{CONTEXTE POLitique ET ÉDUCATIF EN ISRAËL}

Cent ans de conflit non résolu ont entraîné d'importantes souffrances chez les habitants de l'État d'Israël. L'avenir incertain d'un monde aux prises à des tensions mondiales croissantes ne laisse pas beaucoup de place à l'espoir. Pourtant, même dans ces régions perturbées, beaucoup considèrent que l'éducation offre une voie pour apaiser les conflits et favoriser la reconnaissance mutuelle et la réconciliation.

\footnotetext{
* Article traduit par Sylvaine Herold.
} 
Le conflit israélo-palestinien trouve son origine dans le début de la colonisation sioniste de la Palestine, revendiquée par les Juifs vers la fin du $\mathrm{XIX}^{\mathrm{e}}$ siècle comme étant leur terre d'origine. Ce conflit, en apparence inextricable, est la conséquence d'au moins deux discours idéologiques dominants (l'un juif, l'autre palestinien) sur le contrôle de la terre et la reconnaissance de la souveraineté du groupe. Historiquement, la région n'a jamais été autonome et se trouve marquée par une longue histoire de domination coloniale et impériale. Deux événements historiques majeurs, avant la création de l'État d'Israël en 1948 - à savoir la dissolution de l'Empire ottoman, qui a contrôlé la Palestine pendant quatre siècles, et la montée de l'antisémitisme qui a culminé avec l'Holocauste de la Seconde Guerre mondiale - permettent de situer ce conflit dans un contexte international plus vaste. La guerre de 1948, appelée guerre d'Indépendance par les Israéliens et Naqbe (la Catastrophe) par les Palestiniens, a constitué le premier affrontement militaire ouvert entre les mouvements nationalistes sioniste et palestinien. Les Palestiniens sont une minorité autochtone en Israël qui représentait, jusqu'en 1947, les deux tiers de la population de la Palestine.

De nos jours, le conflit judéo-palestinien demeure le conflit le plus explosif d'Israël, et il oppose sans relâche la majorité juive ( $80 \%$ de la population) à la minorité palestinienne (principalement musulmane). Bien qu'il existe une forte asymétrie structurelle entre les deux communautés, celles-ci sont toutes deux convaincues de disposer du monopole de la vérité objective concernant le conflit et l'identité de l'agresseur, ce qui mine toute possibilité de résolution du conflit.

Depuis sa création, l'État d'Israël s'est engagé à assurer une pleine égalité politique et sociale à l'ensemble de ses citoyens, quelle que soit leur religion ou leur appartenance ethnique ${ }^{1}$. Pourtant, le gouvernement israélien a, dans l'ensemble, mis en œuvre des politiques ségrégationnistes à l'égard des minorités non juives.

Ces conflits se reflètent également dans le système éducatif israélien, lequel est divisé en différents secteurs éducatifs : secteurs juif non religieux, juif religieux national, juif orthodoxe, druze et arabe, tous sous l'égide du ministère de l'éducation israélien. Au sein de cette offre éducative diverse, on attend des établissements scolaires qu'ils n'exercent pas de discrimination sur quelque fondement que ce soit, conformément à la législation en vigueur.

En Israël, les initiatives en faveur de l'intégration ont commencé avec l'approbation en 1968 du rapport Rimlat, qui a défini les grandes lignes directrices de la première réforme de l'intégration mise en ouvre par l'État. Cette réforme a restructuré les niveaux élémentaires et secondaires du système éducatif, en remplaçant le modèle binaire (8-4) par un modèle qui consiste en six années d'école élémentaire, trois années de secondaire inférieur et trois années de

1. Une loi fondamentale votée le 19 juillet 2018 par le Parlement israélien définit Israël comme « le foyer national du peuple juif. La langue arabe perd son statut de langue officielle au profit d’un « statut spécial ». 
secondaire supérieur. Les promoteurs de cette réforme supposaient en effet que l'égalisation des opportunités éducatives ne pouvait se faire dans un système scolaire divisé selon des lignes de partage ethniques, sociales et économiques.

Le périmètre de l'intégration s'est trouvé dans les faits fortement réduit : d'une part car on en a exclu les enfants palestiniens inscrits dans des filières religieuses ultra-orthodoxes juives, indépendantes de l'État; d'autre part du fait de l'opposition croissante des municipalités et des parents des communautés les plus aisées, qui craignaient que la réforme ne compromette les intérêts de leurs enfants. Ainsi, seuls $56 \%$ des enfants juifs étaient inscrits dans des établissements dits intégrés à la fin des années 1970, période qui a marqué le point culminant des efforts de mise en œuvre de la réforme de l'intégration.

L'inclusion des élèves à besoins spéciaux au sein du système éducatif standard constitue un autre aspect des initiatives en faveur de l'intégration. La Loi sur l'éducation spéciale de 1988 stipule clairement que ces élèves doivent être placés dans un contexte éducatif standard. Le ministère de l'éducation a depuis investi d'importantes ressources et a consenti à d'importants efforts afin de promouvoir l'inclusion, via des directives officielles et des dispositifs administratifs. L'adoption de cette politique a conduit au transfert d'un nombre important d'élèves handicapés dans des établissements et des classes standards, seuls les élèves en sévère situation de handicap continuant à être scolarisés dans des configurations plus spécialisées. En 2013, on dénombrait 198400 élèves à besoins spécifiques en Israël, dont 59,8 \% suivaient une scolarité générale, 21,9 \% étaient scolarisés en classe spéciale dans des établissements standards et 18,3\% dans des établissements spécialisés et des jardins d'enfants.

Comme cela a pu être constaté dans d'autres pays occidentaux (par exemple, aux États-Unis, en France ou au Royaume-Uni), où les chercheurs se montrent de plus en plus critiques à l'encontre non pas des fondements de l'intégration mais de la manière dont elle est mise en œuvre, les efforts d'intégration en Israël, que ce soit en faveur des minorités ethniques ou des élèves à besoins spécifiques, ont essuyé de nombreux revers. La première réforme de l'intégration est tombée en disgrâce dans les années 1980 et les principaux fondements et idéaux de cette réforme ont progressivement été sapés par d'autres politiques et mini-réformes.

Les propositions de réforme éducative plus récentes (par exemple, le rapport Dovrat, 2005) reflètent les processus profonds à l'œuvre depuis trente ans dans la société israélienne, sous l'influence des évolutions idéologiques et politiques mondiales. La société israélienne offre en effet un exemple flagrant de l'influence croissante de l'idéologie néolibérale, laquelle appelle à la refondation des liens entre l'État et la société. La déségrégation tournée vers l'intégration ne constitue dès lors pas nécessairement une réponse adaptée aux problèmes d'une société diverse et conflictuelle. Car l'histoire de l'intégration montre que, même lorsqu'elles sont initiées par l'État, les politiques d'intégration sont complexes à mettre en œuvre, difficiles à soutenir et plus difficiles encore à maintenir. 
La discrimination éducative existant à l'égard de la minorité arabopalestinienne a fait l'objet de nombreuses études en Israël. Une étude récente révèle par exemple que seuls 5600 élèves arabo-palestiniens fréquentent de nos jours une école située dans le secteur juif et 360 élèves juifs une école dans le secteur arabe. Si Juifs comme Palestiniens considèrent que la séparation est efficace, une question demeure cependant, qui a tourmenté plus d'un système scolaire national : séparer est-il nécessairement synonyme d'inégalité ?

Si l'on compare les deux systèmes, on constate d'importants écarts en termes d'équipements, de qualification des enseignants, de taux de rétention des élèves et de niveaux de services spéciaux. En termes de réussite, bien que la part d'élèves passant les examens de fin d'études secondaires dans le secteur arabe soit similaire à celle du secteur hébraïque et que l'écart entre les élèves juifs et palestiniens obtenant le certificat de fin d'études se soit réduit (de $17 \%$ en 2000 à $12 \%$ en 2015), les écarts de réussite demeurent importants, quand il s'agit du niveau de difficulté de l'examen d'anglais de fin d'études (niveau 5 unités) ${ }^{2}$ : dans le secteur hébraïque, $58 \%$ réussissent l'examen, contre $14 \%$ dans le secteur arabe et $23 \%$ dans le secteur druze.

Séparer les élèves selon leur secteur ethnique d'origine a au moins un avantage évident : l'enseignement peut se faire en arabe dans les établissements du secteur arabe. Mais cela comporte également de nombreux inconvénients. Le secteur arabe ne dispose pas du même degré d'autonomie que le secteur juif pour déterminer les priorités éducatives. Les établissements du secteur arabe sont surveillés de près par les autorités et ne sont pas libres d'enseigner aux élèves le récit historique palestinien, car la plupart des supports éducatifs sont simplement traduits à partir du curriculum hébraïque. De nombreux enseignants sont en outre pris dans un conflit de loyauté entre leur employeur, le ministère de l'éducation, et leur communauté palestinienne.

Les effets de la ségrégation sont également visibles dans les programmes scolaires. Tandis que les programmes juifs mettent l'accent sur des contenus d'enseignement liés à la nation juive et à sa construction, les programmes palestiniens sont épurés de tout contenu national palestinien. Ainsi, les élèves juifs sont-ils incités à s'engager dans le projet collectif de la nation juive, tandis que les élèves palestiniens doivent accepter la définition d'Israël comme État juif et démocratique.

À bien des égards, le système éducatif israélien aggrave les cassures ethniques et religieuses et perpétue les divisions qui caractérisent dans son ensemble la société israélienne. Les politiques éducatives sont utilisées comme des mécanismes de contrôle afin de sécuriser l'hégémonie culturelle juive,

2. Lors de l'examen de fin d'études secondaires israélien (Bagrout), les élèves passent des examens dans plusieurs matières obligatoires, dont l'anglais. Dans la plupart des matières, les examens sont disponibles à différents niveaux de difficulté, exprimés en « unités d'étude » (5 unités d'étude correspondant au niveau de difficulté le plus élevé), et les élèves peuvent choisir le nombre d'unités dans lesquelles ils sont testés, sous réserve d'obtenir le total d'unités d'étude requis pour l'obtention du diplôme. Source : Jewish Virtual Library. (NdT) 
conformément à la définition d'Israël comme État juif, en cultivant un ethos sioniste chez les élèves juifs et un sentiment d'infériorité chez les citoyens palestiniens.

\section{L'ÉDUCATION POUR L'INTÉGRATION ET LA RÉCONCILIATION}

Dans ce contexte, des rencontres intergroupes ont été organisées dès le début des années 1950. Les rencontres à visée spécifiquement éducative, conçues pour dépasser l'hostilité et contribuer à la coexistence, n’ont été initiées que dans les années 1980 (Helman, 2002), en réaction à la publication d'une enquête révélant l'existence d'attitudes anti-démocratiques parmi la jeunesse juive à l'égard de la minorité palestinienne. La publication de cette enquête a attisé les craintes de voir un jour la société israélienne rejeter son caractère démocratique (Katz et Kahanov, 1990) et a entraîné la formation de ce que Rabinowitz (2000) appelle le "secteur de la coexistence », qui est axé sur le développement d'activités en faveur de l'élaboration de la coexistence.

Au cours des années 1990, les dialogues de coexistence portant sur les relations conflictuelles entre groupes et sur la possibilité de la réconciliation ont été menés, pour l'essentiel, par des ONG et se sont vus renforcés par les Accords d'Oslo (1993-1995). Ces initiatives, allant de simples réunions ponctuelles à des processus de dialogue plus continus, ont concerné une grande variété d'acteurs : jeunes, étudiants, professeurs d'université, autres professionnels.

Ces rencontres réunissaient généralement huit à douze participants de chaque nationalité. Elles étaient menées par un facilitateur juif et un facilitateur palestinien et avaient lieu dans le cadre d'organisations éducatives et communautaires. S'il a été reconnu que ces rencontres pouvaient constituer une stratégie de changement personnel et social, leur efficacité a néanmoins été remise en cause.

On peut distinguer quatre principaux modèles de rencontres : le modèle de la coexistence, le modèle des projets communs, le modèle de la confrontation et le modèle du story-telling, lesquels offrent tous des avantages et des inconvénients potentiels, notamment quand il s'agit de la possibilité de se sentir inclus en tant que participant, autorisé à exprimer ouvertement son point de vue et rassuré quant au fait que la participation à ces rencontres contribuera, d'une manière ou d'une autre, à faire émerger des solutions possibles. Le modèle de la coexistence insiste sur les ressemblances interpersonnelles ( Nous sommes tous des êtres humains»), les similarités culturelles et la notion de coopération. Le modèle des projets communs repose sur l'hypothèse selon laquelle œuvrer ensemble à un objectif commun supérieur réduit l'hostilité entre les groupes, favorise la coopération et l'émergence d'une identité commune capable de transcender l'identité distinctive de chaque groupe. Le modèle de la confrontation met l'accent sur les relations de pouvoir existant entre les différentes parties au 
conflit, dans l'objectif d'autonomiser les membres minoritaires et de leur permettre de se confronter à la majorité, à travers des discussions sur les identités nationales, les ambitions nationales et civiles, la discrimination. Le quatrième et dernier modèle combine les aspects de coexistence et de confrontation entre les groupes en adoptant une approche narrative, dans laquelle les participants de chaque groupe s'engagent dans une mise en récit et partagent leurs histoires, leurs expériences et leurs souffrances, personnelles et collectives, liées au conflit.

Plus récemment, les initiatives éducatives ont déplacé le travail éducatif de la sphère de l'éducation intégrée informelle et alternative à la sphère formelle. En 1984, le premier établissement scolaire intégré bilingue judéo-palestinien a été créé à Neve Shalom/Wahat Al-Salam³ . Depuis 1998, d'autres organisations ont rejoint ce mouvement (initiatives "Hands in Hands » et "Hagar ») et il existe aujourd'hui sept établissements scolaires judéo-palestiniens intégrés bilingues en Israël, accueillant une population d'environ 1200 élèves. Un seul de ces établissements propose une scolarité complète (des niveaux K1 à K12) ${ }^{4}$.

Ces établissements sont reconnus et soutenus par le ministère de l'éducation israélien. Ils suivent le curriculum standard du système scolaire laïc d'État, curriculum complété à la marge afin de refléter l'engagement idéologique de ces établissements en faveur de l'égalité et de la coexistence. Les fêtes religieuses de chaque groupe sont pour la plupart reconnues, ainsi que leur récit national respectif. Des activités culturelles représentant tous les groupes sont en outre intégrées aux programmes des établissements. Ces établissements sont exposés au risque de voir certains de leurs engagements limités par le ministère de l'éducation, comme par exemple l'étude du récit historique arabo-palestinien.

Comme c'est le cas dans d'autres établissements spéciaux reconnus par l'État, les fonds alloués par le ministère de l'éducation s'avèrent insuffisants pour doter les établissements intégrés bilingues du matériel et du personnel supplémentaires dont ils ont besoin. Ils sont donc contraints de facturer des frais de scolarité aux familles qui y inscrivent leurs enfants; ce qui explique sans doute que les familles attirées par les établissements bilingues, juives ou palestiniennes, appartiennent pour l'essentiel aux classes moyennes et supérieures.

L'une des caractéristiques principales de ces établissements intégrés est le co-enseignement qu'ils proposent, la présence d'un enseignant juif et d'un enseignant palestinien dans chaque classe étant censée favoriser le bilinguisme. Mais, en raison du coût très élevé de ce dispositif, il a été décidé de limiter le co-enseignement aux matières enseignées par les enseignants titulaires ; l'enseignement des autres matières, telles que les sciences et l'art, est dispensé par un seul enseignant, issu de l'un ou l'autre des groupes ethniques.

3. Village fondé en 1969 entre Jérusalem et Tel-Aviv-Jaffa et dont le nom signifie « Oasis de paix ». Ses habitants juifs et arabes œuvrent pour l'égalité de droits et l'entente entre les deux peuples. Voir : http://wasns.org/ (NdIR) 4. Dans de nombreux pays, le système K-12 désigne la scolarité obligatoire de 6 à 18 ans, après le Kinderdergarten (K - équivalent de la maternelle). Les classes sont appelées des « grades », soit K 1 à K12. (NdIR) 
L'engagement de ces établissements, à la fois en faveur de la réussite académique de leurs élèves, telle que mesurée par les examens nationaux obligatoires, et de leurs objectifs propres en matière de bilinguisme et de coexistence, représente par ailleurs un délicat exercice d'équilibriste. Une multitude de facteurs contextuels exerce des pressions incessantes sur ces établissements, ce qui oblige leurs administrateurs - et, de fait, tous les participants - à adopter une attitude d'évaluation dynamique continue, afin d'équilibrer des objectifs qui peuvent ne pas toujours être compatibles.

L'initiative éducative la plus récente en matière d'intégration est le Programme d'éducation partagée (Shared Education Program) développé, depuis 2012, par le ministère de l'éducation et des ONG partenaires dans des zones mixtes d'Israël, sur le modèle de ce qui a été fait en Irlande du Nord. Ce programme propose des formations pour les enseignants, développe des méthodologies et des matériels d'apprentissage, en face-à-face ou en ligne, et recense les activités bénéficiant du soutien des autorités locales. L'approche en termes d'éducation partagée est conçue pour permettre le développement de partenariats à long terme entre les établissements juifs et palestiniens, en axant ces partenariats sur un travail collaboratif intégré à différents domaines d'étude. On attend de ce programme qu'il procure des avantages éducatifs aux apprenants grâce à l'utilisation efficace des ressources et au soutien de la réalisation d'objectifs sociaux, tels que l'égalité des chances, le respect de la diversité et de la solidarité, tout en préservant l'identité de chaque groupe.

Enfin, il convient également de mentionner d'autres initiatives qui mettent l'accent sur des approches éducatives actives plutôt que cognitives - à travers le sport, l'art et l'activisme social. Le programme "Sadaka-Reut » a été créé en 1983 dans le but d'éduquer et de responsabiliser la jeunesse ainsi que les étudiants juifs et palestiniens, à travers des programmes uni et binationaux, afin de poursuivre activement l'objectif de changement social et politique grâce à des partenariats binationaux. "Peace Child Israel» a été fondée en 1988 afin de promouvoir la coexistence par le biais du théâtre et des arts. Les programmes de cette organisation rassemblent pendant huit mois des adolescents juifs et palestiniens issus d'établissements scolaires israéliens, lors de rencontres hebdomadaires, et aboutissent à la création de pièces originales traitant de la coexistence et de ses défis. Depuis 2001, «Football 4 Peace » [Le football pour la paix] se sert du sport pour offrir des formations centrées sur les valeurs, qui visent à promouvoir le respect, la responsabilité, l'inclusion, la neutralité et l'égalité.

\section{POSTUlats THÉORIQUes}

Quel que soit leur contexte organisationnel, toutes ces initiatives revendiquent différents postulats théoriques, dont les plus importants sont examinés dans ce qui suit. 
L' « hypothèse du contact » constitue notamment la base de la plupart des initiatives éducatives d'intégration. Selon cette hypothèse, promouvoir le contact entre les membres de différents groupes ethniques permettrait de réduire les tensions, ce qui se traduirait par des attitudes plus positives et plus tolérantes. Elle suggère que le contact entre les groupes - quand il a lieu dans des conditions d'égalité et d'interdépendance favorisant une interaction soutenue et l'amitié entre les participants, dans des contextes légitimés par un soutien institutionnel permet d'atténuer le conflit et les attitudes négatives entre les groupes.

Les théories de l'apprentissage social suggèrent en outre que les attitudes à l'égard des groupes extérieurs sont façonnées par l'information et la connaissance acquises à partir des contextes sociaux immédiats et des multiples canaux médiatiques. Les formations interculturelles et l'information anti-préjugés sont dès lors considérées comme des moyens efficaces pour lutter contre les généralisations négatives.

Les théories du développement cognitif social considèrent par ailleurs que le comportement des enfants dépend du stade de développement de leurs compétences cognitives; la recherche dans ce champ s'oppose en outre à une compréhension unidirectionnelle du développement des stéréotypes, en ancrant ceux-ci dans des variables complexes, liées aux contextes sociaux et aux relations avec autrui, qui rendent certaines relations conflictuelles entre les groupes et les identités de groupes particulièrement saillantes ou qui mettent l'accent sur l'application universelle des principes moraux d'équité et d'égalité.

Les théories psychologiques apportent certaines réponses à la question du développement des préjugés, en suggérant la dépendance socio-contextuelle et socio-cognitive de ceux-ci, tout en montrant que les préjugés peuvent être réduits par la promotion du contact entre les groupes, d'identités communes et de normes sociales inclusives, de formations aux compétences socio-cognitives, du raisonnement moral et de la tolérance.

\section{RÉSULTATS DE RECHERCHES}

Les résultats des recherches menées sur les effets des initiatives éducatives d'intégration en faveur de la réconciliation en Israël sont pour l'heure limités et provisoires.

Les chercheurs ont constaté que les jeunes des deux groupes arrivaient aux rencontres avec une connaissance limitée les uns des autres et des préjugés réciproques négatifs. Dans ce contexte, les recherches ont montré que les rencontres ont permis aux jeunes d'améliorer leurs perceptions réciproques initiales, ce qui semble indiquer que des pratiques transformatives peuvent s'avérer efficaces, en dépit d'un contexte sociopolitique difficile.

Des contextes similaires ont également été examinés par les chercheurs, afin d'étudier les changements différentiels possibles dans les croyances centrales et périphériques, ainsi que la pérennité de ces effets. Ces études montrent que 
les programmes éducatifs de réconciliation peuvent influencer efficacement les attitudes et croyances périphériques des jeunes.

Des études qualitatives récentes donnent néanmoins moins de raisons d'être optimiste. Des recherches menées auprès d'étudiants participant à des rencontres intergroupes ont révélé la prédominance des Juifs parmi les participants aux dialogues judéo/arabo-palestiniens. D’autres études ont examiné les ressources discursives mobilisées par les participants lors de ces rencontres et ont observé que la rhétorique nationale en termes de majorité-minorité est influencée par le contexte de l'État-nation et semble guider les échanges de ces rencontres, imposant ainsi un cadre essentialiste aux identités ethniques/ nationales des participants.

Une étude comparative des programmes "Peace Child» et «SadakaReut » a conclu que les programmes éducatifs les plus à même de favoriser une participation durable aux activités de changement social sont ceux qui offrent aux participants la possibilité d'examiner de manière critique le rôle de leur propre groupe dans les contextes de conflit - notamment parmi les membres des groupes dominants -, tout en fournissant un environnement structuré pour que les membres des différents groupes interagissent.

Une enquête menée dans treize cours d'apprentissage-action régulièrement accrédités dans onze établissements d'enseignement supérieur israéliens a révélé que, bien que les participants à ces cours soient conscients des inégalités existant en Israël et que ces cours leur offrent la possibilité d'une réflexion critique, ils ont tendance à s'auto-définir comme apolitiques et sont réticents à s'engager dans des discussions ou de l'activisme politiques. L'étude conclut en insistant sur l'importance de la culture institutionnelle dans laquelle ont lieu ces activités éducatives, en suggérant que, puisque ni la faculté ni les étudiants ne sont prêts à remettre en cause le passage sous silence hégémonique des questions politiques relatives au conflit dans les établissements universitaires israéliens, la plupart des bénéfices potentiels de ces programmes en sont perdus.

Les recherches menées dans les établissements intégrés bilingues mettent en lumière les difficultés auxquelles doit faire face l'enseignement bilingue, biculturel et binational, dans sa tentative de respect des différences, de culture $\mathrm{du}$ dialogue et d'inspiration d'une vision morale. Ces recherches montrent en outre que les contextes politiques sont des éléments cruciaux pour comprendre les initiatives éducatives transformationnelles.

Ces vingt dernières années, une recherche longitudinale globale portant sur l'enseignement judéo-palestinien bilingue montre que les réalités sociales contrastées des Juifs et des Palestiniens influencent les motivations des familles à inscrire leurs enfants dans les établissements intégrés judéo-palestiniens, tout comme les statuts différents de l'hébreu et de l'arabe dans la société israélienne influencent les motivations de chaque groupe à acquérir la langue de l'autre.

Les données ethnographiques rassemblées suggèrent en outre que la question de l'identité nationale et des récits historiques est devenue le défi 
éducatif principal pour les parents comme pour le personnel enseignant. Les tensions sont visibles côté palestinien, en particulier parmi les enseignants, qui se considèrent comme à l'avant-garde de la lutte pour la sauvegarde du récit national palestinien, lequel demeure non reconnu par les autorités éducatives israéliennes. La plupart des Juifs libéraux considèrent que l'expression d'une identité culturelle et religieuse israélo-palestinienne à l'école est légitime. Mais l'identification nationale à l'Autorité palestinienne est mal accueillie, tout comme les points de vue qui cherchent, de quelque façon que ce soit, à renier le droit d'Israël à être un État juif.

Selon une récente étude sur les diplômés de l'école israélienne, dont les résultats sont conformes à ceux de recherches plus récentes sur les initiatives éducatives bilingues intégrées, on observe un favoritisme intragroupe moindre chez les élèves juifs et palestiniens diplômés, ainsi que des perceptions accrues de similarités intergroupes.

\section{Perspectives CRItiques}

Nombre de chercheurs ont exprimé leur scepticisme quant à l'impact possible des rencontres intergroupes entre Juifs et Palestiniens. Si ces rencontres peuvent effectivement avoir des effets à court terme qui modifient les comportements intergroupes, elles semblent avoir échoué à influencer les attitudes de la société dans son ensemble. Cette absence d'influence serait liée au temps d'exposition trop court entre participants permis par la plupart de ces rencontres, au manque de suivi de celles-ci, mais également au fait que la participation y est généralement volontaire - les opposants à la réconciliation n’y participant donc vraisemblablement pas.

Ces rencontres sont en outre, la plupart du temps, déconnectées de la vie quotidienne des participants. Elles s'intéressent principalement aux questions de culture, d'identité ou aux problématiques interpersonnelles et elles évitent d'aborder les profondes asymétries structurelles et institutionnelles existant entre les groupes. Enfin, le fait que la position des Juifs et des Palestiniens diffère au sein de ces rencontres et que ces dernières puissent donc affecter différemment les deux groupes est passé sous silence. Il en résulte que les leçons apprises lors de ces ateliers de dialogue ne sont généralement pas appliquées de manière substantielle dans la vie courante.

Aux limites mentionnées précédemment, nous souhaiterions en ajouter d'autres, plus fondamentales. Ces initiatives éducatives en faveur de la coexistence manquent encore de théorisation pédagogique; à ce jour, leurs objectifs sont définis sur la base de perspectives fonctionnelles, psychologiques et idéalistes. Ainsi conçues, ces initiatives éducatives reflètent les conceptualisations modernes totalisantes de l'Occident et situent le foyer de la maladie dans l'esprit individuel, qui doit être traité. Le traitement préconisé devrait, qui plus est, dans la plus pure tradition positiviste psychologique, être universellement appliqué à des 
individus solipsistes, et faire fi des facteurs historiques et contextuels. Mais une question se pose : travailler à partir des postulats théoriques constitutifs du monde occidental moderne, qui a vu éclore nombre des conflits que l'éducation en faveur de la coexistence entend aider à atténuer et à surmonter, constitue-t-il vraiment la base adéquate pour bâtir cet enseignement?

Lorsque l'éducation en faveur de la réconciliation est considérée partout comme étant hautement désirable, elle cesse d'être pertinente et perd de son influence potentielle car, en présentant ses valeurs comme des évidences universelles, on occulte ce qui est fondamental dans le conflit - les multiples représentations de la vérité, les diverses compréhensions de la justice - et, plus important encore, les liens étroits qui existent entre le conflit, l'ordre capitalistique actuel et la division mondiale du travail. En d'autres termes, cela revient à ne pas prendre en compte les arrangements sociaux qui institutionnalisent l'inégalité et l'injustice, en évitant de répondre à des questions telles que : qui sommes-« nous» ? À quelles perceptions de la justice tenons-nous ? Quel dialogue voulons-nous cultiver et dans quelles conditions? L'éducation en tant qu'idéal universel ne constitue dès lors pas une bonne formule pour favoriser une cohabitation pacifique.

Si l'éducation prend au sérieux les principes qui la sous-tendent - à savoir l'affirmation, la reconnaissance et la réhabilitation de l'altérité -, elle devrait commencer par porter un regard critique sur les certitudes épistémologiques et métaphysiques de la modernité occidentale.

Il semble urgent que les initiatives éducatives inclusives en faveur de la réconciliation réexaminent leurs fondements paradigmatiques et questionnent les structures politiques qui entretiennent les conflits qu'elles tentent de surmonter. Mais même ainsi, il est utile de rappeler que les conflits historiques sanglants auxquels ces initiatives tentent de remédier trouvent davantage leur origine et les raisons de leur persistance dans l'allocation inégale des ressources matérielles que dans l'esprit d'individus perturbés.

\section{POUR EN SAVOIR PLUS SUR LES DIFFÉRENTES INITIATIVES ÉDUCATIVES}

BAR-TAL D., ROSEN Y. (2014) : «Conditions for the Development of Peace Education in Societies Involved in Intractable Conflict ", in D. C. BERLINER et H. KUPERMINTZ (coord.), Fostering change in institutions, environments, and people: a festschrift in honor of Gavriel Salomon, New York, NY: Routledge, p. 231-250.

BEKERMAN Z. (2007) : "Rethinking intergroup encounters: Rescuing praxis from theory, activity from education, and peace/co-existence from identity and culture ", Peace Education, no 4(1), p. 29-41.

BEKERMAN Z., ZEMBYLAS M. (2012): Teaching contested narratives: Identity, memory and reconciliation in peace education and beyond, Cambridge University Press. 
GOLAN D., SHALHOUB-KEVORKIAN N. (2014) : "Community-engaged courses in a conflict zone: a case study of the Israeli academic corpus ", Journal of Peace Education, $\mathrm{n}^{\circ} 11(2)$, p. 181-207.

HUGHES J., LOADER R. (2015) : "'Plugging the gap': shared education and the promotion of community relations through schools in Northern Ireland ", British Educational Research Journal, $\mathrm{n}^{\circ}$ 41(6), p. 1142-1155.

JAMAL A. (2009): The Arab public sphere in Israel: Media space and cultural resistance, Indiana University Press.

MAOZ I. (2009) : «Educating for peace through planned encounters between Arabs and Jews in Israel: A reappraisal of effectiveness ", in G. SALOMON et E. CAIRNS (coord.), Handbook of Peace Education, New Jersey: Psychology Press.

MAOZ I. (2011) : « Does contact work in protracted asymmetrical conflict? Appraising 20 years of reconciliation-aimed encounters between Israeli Jews and Palestinians », Journal of Peace Research, $n^{\circ}$ 48(1), p. 115-125.

PAYES S. (2017) : "Education across the divide: Shared learning of separate Jewish and Arab schools in a mixed city in Israel ", Education, Citizenship and Social Justice, vol. 13, n 1, p. 19-35. [DOI : https://doi.org/10.1177/1746197917698489]

ROSS K. (2015) : "Quality as critique: promoting critical reflection among youth in structured encounter programs. Journal of Peace Education, $\mathrm{n}^{\circ}$ 12(2), p. 117-137. 